Théologiques

Théologiques

\title{
Du bon usage de la mémoire de l'esclavage des Noirs comme un possible capital de rédemption \\ L'exemple de Bakhita
}

\section{Armel Brice Adanhounme}

Volume 13, numéro 2, automne 2005

Violence et souffrance rédemptrices

URI : https://id.erudit.org/iderudit/013608ar

DOI : https://doi.org/10.7202/013608ar

Aller au sommaire du numéro

\section{Éditeur(s)}

Faculté de théologie et de sciences des religions, Université de Montréal

\section{ISSN}

1188-7109 (imprimé)

1492-1413 (numérique)

Découvrir la revue

\section{Citer cet article}

Adanhounme, A. B. (2005). Du bon usage de la mémoire de l'esclavage des Noirs comme un possible capital de rédemption : l'exemple de Bakhita. Théologiques, 13(2), 133-163. https://doi.org/10.7202/013608ar
Résumé de l'article

À partir du cadre méthodologique de la biographie comme théologie (McClendon 1970), cet article propose la mémoire de l'esclavage des Noirs comme un possible capital de rédemption. L'argument est basé sur la vie de sainte Joséphine Bakhita, ancienne esclave africaine, qui, devant mourir, a supplié de desserrer les chaînes si lourdes de l'esclavage. Dans une perspective hagiographique, trois points structurent le paradigme d'une théologie de l'esclavage chez Bakhita : le récit, le souvenir et la récupération de la mémoire de l'esclavage. L'idée de possible capital de rédemption s'articule conséquemment autour des mêmes caractéristiques : la subversion du récit hégélien sur l'esclavage des Noirs par le modèle de Bakhita, une rupture historique et épistémologique de l’interprétation de ce récit, et une rébellion prophétique par la possibilité de dire non.
Tous droits réservés (C) Faculté de théologie et de sciences des religions, Université de Montréal, 2006
Ce document est protégé par la loi sur le droit d'auteur. L'utilisation des services d'Érudit (y compris la reproduction) est assujettie à sa politique d'utilisation que vous pouvez consulter en ligne.

https://apropos.erudit.org/fr/usagers/politique-dutilisation/ 


\title{
Du bon usage de la mémoire de l'esclavage des Noirs comme un possible capital de rédemption L'exemple de Bakhita
}

\author{
Armel Brice Adanhounme \\ Étudiant au programme de Ph. D. en administration \\ HEC Montréal
}

Mon nom: offensé; mon prénom: humilié; mon état: révolté; mon âge: l'âge de la pierre [...]. Ma race: la race tombée. [...] Nous avions bondi, nous les esclaves, nous le fumier, nous les bêtes au sabot de patience. Nous courrions comme des forcenés; les coups de feu éclatèrent... Nous frappions. La sueur et le sang nous faisaient une fraîcheur. [...] Alors, ce fut l'assaut donné à la maison du maître. [...] J'entrai. C'est toi, me dit-il, très calme... C'était moi, c'était bien moi, lui disais-je, le bon esclave, le fidèle esclave, l'esclave esclave, et soudain ses yeux furent deux ravets apeurés les jours de pluie... je frappai, le sang gicla: c'est le seul baptême dont je me souvienne aujourd'hui. (Paroles du rebelle, dans Césaire 1958, 68, 71) 
Et il court et frappe toujours, le forcené esclave que Césaire (1958) et les autres hérauts de la négritude, après les chrétiens, voulaient libérer. Lorsque Hegel (1964, 75-80), se basant sur les comptes rendus prolixes des missionnaires, représentait le Noir de la mentalité prélogique comme "l'homme naturel dans toute sa sauvagerie et sa pétulance", comme le remarquera Eboussi Boulaga $(1999,63)$ un siècle plus tard, il décrivait moins le nègre qu'il ne connaissait guère qu'il prophétisait celui que nous allions devenir: les King Kongs du XXI ${ }^{\mathrm{e}}$ siècle. Une vision apocalyptique, mais en fait anachronique d'une Afrique qui se saborde elle-même et dont Stephen Smith (2003), dernier hégélien de l'anhistoricité du continent noir, a annoncé la "négrologie ». Le présent, écrit-il, n’a pas d'avenir en Afrique, puisque l'Afrique est un «Ubuland sans frontière, terres de massacres, de famine, mouroir de tous les espoirs " (13). Les Noirs auraient pu voir dans l'esclavage atlantique, continuum des esclavages oriental et clanique, la forme outrée de leurs souffrances. Hélas! Mais il suffit que Shiva Naipaul ou autres explorateurs du primitif — les «négrophobes» selon les détracteurs de Smith (par exemple Diop, Tobner et Vershave 2005) — évoquent l'incomplétude de l'Afrique pour que les Africains et les africanistes sortent de leurs gonds. Et pourtant, il y a longtemps que le sang gicle en Afrique.

Déjà, les bagnes coloniaux avaient relayé les chaînes de l'humiliation pour civiliser, au nom de Dieu, l'esclave par nature. Dans une reconduction du mandat colonial, les indépendances ratifieront le droit à l'usage et au pouvoir de la force. Passent les douleurs de l'enfantement liées à l'accouchement des souverainetés nationalistes, car l'indigène, bon enfant de l'ethnologie coloniale, devrait grandir et devenir projet de lui-même! L'histoire, la même histoire de la violence sans fin et sans issue, continue. Il y a une dizaine d'années, sortant de l'ordinaire des massacres, guerres civiles et ethniques qui ponctuent normalement la vie en Afrique noire, les Rwandais, à leur tour, croyaient remporter la palme d'or de la barbarie sur les Angolais, les Libériens, les Sierra Léonais, les Somaliens, etc. Mais ce n'est pas connaître les Congolais, les Soudanais, les Ivoiriens et autres gladiateurs qui s'en viendront vite leur discuter le peloton de tête. La souffrance abjecte et la violence inutile auront-elles, un jour, un sens et une fin en Afrique noire?

De l'impossible prédiction à contenir le déferlement de la violence en Afrique, une froide leçon s'impose. Le génocide, la désintégration de l'État, les manches courtes ou les manches longues pour dire les niveaux d'amputation des bras, les opérations effacer-le-tableau pour signifier les nettoyages 
ethniques ne sont pas imprévisibles. Le vandalisme, la tyrannie et l'incurie des dirigeants africains ici et là, mais aussi la démission collective des Africains n'accréditent-ils pas la thèse d'un afro-pessimisme délétère, mais pas moins sensé ? Comme a pu prophétiser un leader africain de malheur, «nous n'avons pas encore vu le bout du tunnel». Les Noirs peuvent toujours explorer les possibilités du pire. Mais en fait de possibilités, elles sont seulement ambivalentes et les termes du choix sont très clairs, du moins, au regard de Dieu: "Je prends aujourd'hui à témoin contre vous le ciel et la terre: je te propose la vie ou la mort, la bénédiction ou la malédiction.» (Dt 30,19) La vie est encore possible en Afrique noire; une fragile vie dont je me permets, dans cet article, de croire à l'avènement. Comme à Sodome et Gomorrhe (Gn 18-19), quelques individus pourront encore sauver l'Afrique: un Paul Rusesabagina au Rwanda ${ }^{1}$ ou un tel autre bon Samaritain du pays de l'Ubuland, car le Seigneur ne peut supprimer le juste et l'injuste. Il s'en trouvera au moins un seul, comme Joséphine Bakhita ${ }^{2}$, dont la mémoire de l'esclavage, au nom de tous les siens, pourra faire advenir la rédemption pour tous.

J'appelle, en effet, possible capital de rédemption, un bon usage de la mémoire des souffrances africaines, sous ses rudes et cruelles formes d'esclavage abject et ses insidieuses survivances sous toutes les formes de réduction anthropologique du Noir, en vue d'une renaissance africaine après le long chemin de croix. L'idée de possible capital de rédemption explore les capacités de salut dissimulées au cœur même de l'histoire africaine, une histoire de souffrance toujours marquée par l'esclavage. Ces capacités, une fois découvertes, peuvent augurer l'avènement de l'Emmanuel africain, Dieu, plantant finalement sa tente en Afrique. Possible, elle tient de l'offre virtuelle, une proposition évangélique qui, comme une grâce, peut être acceptée ou rejetée. Capital, parce que la prise de conscience de la souffrance peut générer un «fond» de production du bien-être collectif. Ce qui implique enfin la rédemption, parce que la croix n'aura pas le dernier mot, et l'Afrique ressuscitera des profondeurs de l'esclavage pour que les

1. C’est l'épopée d'un Rwandais Hutu, pendant le génocide des Tutsis en avril 1994 au Rwanda, portée à l'écran dans le film Hôtel Rwanda (2004) du producteur Terry George. Ce bon Rwandais, gérant de l'hôtel des Mille Collines, à Kigali, va cacher et sauver de la mort quelques 1280 personnes, Hutus et Tutsis confondus, pendant le génocide. Bref, un Oskar Schindler rwandais!

2. Les références à Bakhita sont prises de sa biographie (voir Dagnino 1992). Tout au long de cet article, les pages référées seront simplement citées dans le texte. 
Africains retrouvent l'image de Dieu corrompue par les vicissitudes de l'histoire. Ma perspective est celle de l'hagiographie comme principe de l'inculturation de la foi, où l'eucharistie du lavement des pieds de l'esclave par le maître subvertit les civilités de la théologie des jouisseurs de ce monde. L'objectif de cet article est justement de subvertir le sens de l'esclavage des Noirs en l'ouvrant au salut. À cette fin, je propose de sauver de l'oubli la prodigieuse mémoire de sainte Joséphine Bakhita (environ 1871-1947), ancienne esclave, africaine et chrétienne, pour en faire l'exemplum d'un usage rédempteur de la mémoire de l'esclavage. Relire l'histoire de Bakhita, sous le palimpseste de l'esclavage des Noirs, pour en construire un nouveau récit africain, peut aider à identifier les possibilités du capital de rédemption. Une fois cette méthode précisée, trois points structureront la constitution du possible capital de rédemption: la subversion pour le récit, le souvenir pour la mémoire, et la récupération pour l'hagiographie.

\section{La méthode: Bakhita comme paradigme}

James W. McClendon (1970), à la recherche d'une éthique du personnage, a postulé que la théologie, comme une nouvelle mode en éthique, doit être au moins une biographie, et la biographie, au mieux, une théologie. Outre la théologie de la fides quarem intellectum des bonnes idées, de simples et ordinaires vies nous révèlent le mystère du royaume de Dieu à partir de leur conviction de foi. Une théologie pratique, en fait. Joséphine Bakhita est un exemple vivant de cette conviction qui fait la théologie. Comme elle exhorta les siens, devant mourir: "Desserrez les chaînes de l'esclavage, elles sont si lourdes» (87), sa vie et son message peuvent et doivent servir de pastorale pour une théologie de l'esclavage, si ce n'est pas l'évangélisation elle-même en Afrique noire.

De l'ordre de la réponse à une crise comme en parle Kuhn (1970), le modèle de Bakhita peut servir de paradigme en ce qu'il réfère à un ensemble de constellations de croyances, de valeurs, de techniques, partagées par les membres d'une communauté donnée (175). Autrement dit, dans la communauté esclavagiste qui est la leur, Bakhita, par l'exemplarité de son expérience, s'offre comme un modèle d'intelligibilité des arcanes du récit africain. Comprendre cette configuration fondamentale de l'esclavage aidera à saisir «la révolution» que Bakhita introduit dans le vécu de l'esclavage. Si les paradigmes présupposent toujours des systèmes de croyances basés sur des postulats ontologiques, épistémologiques et méthodologiques, peut-être que le problème du récit africain de l'esclavage réside dans son 
incapacité à sortir des ornières circulaires de l'interprétation présumée du maître. La vie exemplaire de Bakhita, passant de l'esclavage à la liberté, se propose comme une clé de lecture subversive de ce récit; et en cela, ses présupposés théologiques peuvent informer l'écriture de la nouvelle histoire africaine. La vie de Bakhita est une épiphanie africaine de l'esclavage qui cherche la liberté, et le pape Jean-Paul II, en canonisant l'ancienne esclave le 10 février 1993 à Khartoum, au Soudan, fit d'elle un modèle d'imitation pour les chrétiens.

Chez Bakhita, on s'en rendra compte, l'ontologie ne consiste pas dans la simple affirmation de l'égalité entre les êtres humains, mais dans la difficile acceptation de cette égalité dans la différence. Elle est parfaitement chrétienne à cet égard, car la proposition chrétienne de sainteté ne tient pas de l'égalité entre les frères et les sœurs qui sont des pécheurs, mais de la réponse individuelle, dans la différence, à l'appel de Dieu à être en plénitude. Le chrétien, comme le dit la Lettre à Diognète, doit être différent des autres - les païens. Dans cette différence de valeur, Bakhita fonde son épistémologie. Elle cherche à connaître les mécanismes de l'esclavage, bien au-delà du modèle productif aristotélicien du mode d'acquisition naturelle faisant partie de l'économie domestique, le bien de propriété le plus important et le plus nécessaire (Aristote, Économique, I 4). Ou, plus grave encore, de l'apologie augustinienne qui christianise l'argument païen qu'Aristote avait rationalisé: la condition de l'esclavage est un châtiment du péché; péché dont l'affranchissement est dans la rédemption qui fait de tous des hommes libres (La cité de Dieu, XIX, XV-XVI). Pour Bakhita, l'esclavage naît de l'ignorance (84); aussi se propose-t-elle d'enseigner la liberté du Christ à ceux qu'elle appelle les pauvres malheureux. Bakhita n'est pas seulement une victime, mais comme une «participante passionnée » de l'esclavage, sa méthodologie est contenue dans ses dernières paroles, appelant à desserrer les lourdes chaînes de l'esclavage. Tout un testament pour les Églises chrétiennes et les Africains qui en sont membres! Comment prêcher l'évangile de liberté et de libération dans un contexte où des enfants continuent d'être esclaves? Plus généralement, comment le christianisme, conscient de son héritage et de sa collusion dans l'esclavage des Noirs, peut-il proclamer la rédemption d'une Afrique dont il avait annoncé la damnation historique?

Le postulat d'un bon usage de la mémoire de l'esclavage comme un possible capital de rédemption essaie de répondre à ces questions. En traitant du problème de la souffrance dans l'histoire, cette perspective s'insère 
dans la tradition patristique de l'économie du salut, une pédagogie de la révélation de Dieu dans le bic et nunc de l'Afrique en vue d'un arrangement organique de la diversité. La missio ad gentes de l'Église ne commence-t-elle pas par la subversion des bonnes pratiques juives qui entravaient l'épanouissement de l'esprit de liberté que les païens aussi ont reçu ? Crise qui sera résolue dans le concile de Jérusalem (Ac 15,1-35), augurant ainsi la trajectoire d'une ecclesia semper reformanda. Une perspective de crise - selon son étymologie grecque de krinein ( «séparer» ou «décider») — qui va permettre de subvertir les croyances toutes faites par une exigence critique de repositionnement du récit africain, séparé ainsi des explications officielles, voire orthodoxes. La crise de la liberté devient alors un kairos pour l'Afrique. Cela va sans dire que c'est de subversion théologique dont il s'agit; une perspective que les orthodoxes de la pensée d'une théologie unique pourraient trouver «hérétique ". L'esclavage, au nom de Dieu, n'estil pas la plus grande hérésie contre la liberté ? La subversion apparaît ainsi comme une heuristique qui permet de rétablir la vérité!

\section{La subversion: l'histoire de Bakhita à rebours du récit africain}

Lorsqu'il s'agit de situer les Noirs dans la modernité, la référence historique qui rend compte de leur émergence dans le concert de l'humanité est bien sûr l'esclavage. La découverte du barbare, «cet animal étrange à la face humaine» comme a pu dire Eboussi Boulaga (1968, 4-40), posait à juste titre la question de la reconnaissance efficace de l'homme par l'homme. Et Eboussi de s'interroger sur l'origine du Muntu, habitant d'un recoin en dehors de l'histoire, avant que le démiurge ne l'introduisît sur le chantier du monde. Et non seulement ils étaient introduits les uns aux autres comme des esclaves, mais ils étaient aussi identifiés comme créés pour être des esclaves, à telle enseigne qu'être Noir signifiait être esclave et vice-versa. Depuis lors, le Noir a toujours sué, et d'une sueur de sang. Des damnés de la terre, selon l'expression de Frantz Fanon (1968) qui prédisait, non sans raison, qu'après le temps formel de l'esclavage atlantique, les Noirs auraient «à panser des années encore les plaies multiples et quelques fois indélébiles faites à [leurs] peuples par le déferlement colonialiste » (177). Pour le pape Jean-Paul II (1995) aussi, des décennies après les indépendances, l'Afrique panse toujours ses plaies:

L'Afrique actuelle peut être comparée à l'homme qui descendait de Jérusalem à Jéricho, il tomba entre les mains de brigands qui le dépouillèrent, le 
rouèrent de coups et s'en allèrent, le laissant à demi mort (cf. Lc 10,30-37). L'Afrique est un continent où d'innombrables êtres humains - hommes et femmes, enfants et jeunes - sont étendus, en quelque sorte, sur le bord de la route, malades, blessés, impotents, marginalisés et abandonnés. Ils ont un extrême besoin de bons Samaritains qui leur viennent en aide. (41)

L'Afrique de la violence est celle de l'esclavage — l'institutionnalisation du droit à l'usage de la force. Selon l'historien (par exemple Deschamps 1971, 315), le Noir souffre de ce qu'on a dit de lui qu'il est "esclave par nature». L'homme blanc a réussi à faire accepter par la conscience du Noir qu'il est esclave. C'est ainsi qu'à la fin de la traite atlantique correspond la naissance de thèses pseudo-scientifiques et mystiques sur les déficiences ontologiques des Noirs et l'élaboration théorique du racisme européen (Drescher 1992, 361-396). Cheikh Anta Diop a pu écrire que «c'est l'esclavage, au sens occidental, qui a fait de Toussaint Louverture un Prométhée » $(1967,187)$. Seule la force pouvait introduire les sauvages dans la civilité (par exemple De Montesquieu 1952; Berlin et Morgan 1993). Émancipés des tutelles coloniales, les Africains ne vont pas pour autant changer de statut ontologique. L'abolition des travaux forcés n'a pas donné lieu à une nouvelle écriture du récit africain. Les indépendances seront la ratification des régimes de l'hétéronomie (Eboussi Boulaga 1993). D'où la question que reprend l'historien africain, embarrassé: «Quand, comment et par qui le Noir cessera-t-il d'être un nègre? " (Quenum 1993, 298) Sur l'identité contemporaine du Noir, Mbembe (2000), qui se demande si l'Africain peut dire sans réserve «je suis un ex-esclave ", nous éclaire sur l'actualité de l'esclavage en Afrique:

Qui donc est l'esclave sinon celui qui, en tous lieux et en tout temps, possède sa vie, ses biens et son corps comme des choses qui lui sont étrangères? Posséder sa vie et son corps comme des choses étrangères à soi suppose que ce corps et cette vie soient comme une matière extérieure à celui qui les porte et leur sert de charpente. Dans ces conditions, l'on peut attenter au corps, à la vie et au travail de l'esclave. La violence ainsi perpétrée est supposée ne point l'atteindre immédiatement comme réelle et comme présente. Tel étant le cas, « esclave» est le prénom qu'il nous faut donner à celui ou à celle dont il n'est pas interdit de dégrader le corps, de mutiler la vie et de dilapider le travail et les ressources. $(2000,265)$

Le droit de propriété définit toujours l'esclavage dont les formes contemporaines de servitude ont simplement changé (par exemple Bequelle et Myers 1995). Le trafic et le travail des enfants en Afrique de l'Ouest 
aujourd'hui en sont une forme exemplaire (Agossou 2000). Ces survivances justifient ainsi la remarque que faisait Lovejoy $(1983,159)$, à savoir qu'en Afrique de l'Ouest, et plus particulièrement tout au long des côtes de la Sierra Léone, du Ghana et du golfe du Bénin, l'esclavage a survécu dans des institutions qui affectent l'organisation de la société. Bref, il existe une anthropologie de l'esclavage que Claude Meillassoux (1986, 43-67) situe en Afrique sahélo-soudanaise où le développement de l'esclavage est à la fois ancien et exemplaire. De l'Afrique de l'Ouest au Soudan, l'esclavage a laissé de profondes séquelles d'exploitation et de puissants préjudices à telle enseigne que sa description actuelle s'inscrit encore dans le récit que fait Bakhita de sa servitude. Mais Bakhita va plus loin, car l'enjeu ne se réduit pas seulement à décrire, voire décrier, l'esclavage: il faut en subvertir le sens.

La vie de Joséphine Bakhita nous est racontée dans un émouvant récit par sa consœur canosienne Maria Luisa Dagnino, sous le titre Bakhita raconte son histoire (1992). Plus qu'une biographie, il s'agit d'une hagiographie ad aedificationem, comme il est d'usage dans le genre de panégyrique. Voilà pourquoi on doit pouvoir dépasser une lecture biographique du récit pour en faire une relecture théologique. L'intéressée n'avoue-t-elle pas: "C'est à l'invitation de la Révérende Mère Supérieure [...] que je vais raconter quelques épisodes de mon esclavage. Que leur souvenir puisse me faire apprécier, de plus en plus le grand don que le Bon Dieu m'a fait en me choisissant comme son épouse» (33). Deux points sont à retenir dans le récit de Bakhita. Ce sont, d'abord, l'aliénation et l'abjection qui consistent à déformer un être humain, un sujet en objet, et ensuite, la conscientisation et l'humanisation qui est le recouvrement de la subjectivité perdue par un processus de libération ${ }^{3}$ - ou de guérison pour employer un terme chrétien. Le processus d'abjection de l'être humain se découpe en trois étapes: la violence du kidnapping et du traitement (le corps), l'impératif du travail forcé (le travail) et le déni de l'humanité (la vie). Comme être humain reconnu, l'humanisation, en retour, suit les étapes du recouvrement de la mémoire (contre la violence), l'affirmation de l'humanité (la possibilité de dire non) et la vie comme un être humain (dans un engagement libre au service des autres). La relecture de la vie de Bakhita reprend tous ces épisodes et en cela précisément elle est un modèle pour ses frères et sœurs qui en

3. J'emprunte ces concepts au répertoire de la pédagogie de l'oppressé de Paulo Freire (voir 1970; 1998). 
sont restés à l'abjection de l'esclavage sans pouvoir capitaliser celui-ci en instrument de rédemption.

La subjugation d'un être humain, même si elle consiste à faire de celuici un objet, un animal domestique, n'est pas dénuée de raison. Le statut d'abjection présuppose un discours, l'intention d'un acte raisonnable comme le montrent les apologies aristotéliciennes et augustiniennes de l'esclavage. Bakhita récapitule cette histoire de son continent. Elle fut kidnappée par des Arabes, vendue à un général Turc, revendue quatre fois, et finalement achetée par un Italien. Le crime, dit un historien africain au sujet de l'esclavage, concerne l'islam, le christianisme et l'Afrique (Mveng $1963,123)$. Il s'en est suivi la ratification d'une culture de forçats, de violence et de prédation que le colonialisme va légaliser (Mbembe 1996; Samarin 1989). La vérité historique est que les Arabes ont ouvert le marché du peuple noir, et qu'ils seront relayés par les chrétiens qui vont continuer la déportation, avant que les Africains eux-mêmes ne reviennent à la charge. L'imitation des Africains, outre les logiques domestiques de l'esclavage «lignagé » traditionnel (Grace 1975; Memel-Fote 1990), reproduit le règne de l'abjection dans le trafic des caravanes de Noirs capturés au sud du Soudan ou en Mauritanie et convoyés au Yémen ou aux Émirats arabes (Hélène 1997; Daddah 1998). La violence reste la règle du processus d'abjection et le corps de l'esclave est cet objet de violence.

Et ainsi commence le récit de Bakhita, lorsqu'elle fut kidnappée à l'âge de neuf ans par deux trafiquants, et forcée de quitter Ologossa, son village situé dans le Darfour, Bilad es-Soudan, c'est-à-dire le pays des Nègres, désigné comme tel par les géographes depuis le Moyen Âge (16). Elle fut capturée comme un animal, convoyée comme une marchandise et maltraitée. Dans ses propres mots, un esclave est un objet qui appartient à un maître. Tout va alors changer dans sa vie. Son identité devient inconnue. Ce sont ses premiers maîtres qui lui donnent le nom de Bakhita — «Rappelle-toi, désormais, tu t'appelleras Bakhita: Oublie ton nom» (14). L'esclavage s'accommode toujours de l'oubli, et lutte pour la mémoire de l'oubli. Mais le nom d'emprunt est prémonitoire d'un trésor de rédemption, car Bakhita signifie «fortune» en arabe.

La déshumanisation s'achève par l'animalisation, par sévices et châtiments (Ela 1998, 92). Le refus ontologique de l'humanité de l'esclave va entraîner sa paupérisation anthropologique, comme un état d'incomplétude qui affecte sa condition humaine dans ses racines les plus profondes et ses droits fondamentaux (Mveng 1992). Le théologien explique cette 
indigence d'être comme suit: lorsque les personnes sont privées de leur identité, de leur dignité, de leur liberté, de leur pensée, de leur histoire, de leur langage, de leur univers de croyance et de leur créativité fondamentale et de tout leur droit, toute espérance et toute ambition, leur pauvreté devient anthropologique (Mveng 1994). Mais Bakhita ne reste pas, à la différence de ses congénères esclaves, une pauvre anthropologique: elle se refait à l'image de Dieu, en recouvrant la mémoire de l'esclavage, transfigurant ainsi un événement historique en un avènement théologique. Entre l'abjection et l'humanité, les deux voies possibles de la pauvreté anthropologique, Bakhita nous apprend comment l'on peut redevenir sujet. C'est cela l'humanisation par la conscientisation, catégorie du bon usage de la mémoire de l'esclavage qu'il faut maintenant saisir.

\section{Le souvenir: du bon usage de la mémoire chez Bakhita}

Une interprétation judicieuse de l'esclavage n'est possible que par un pertinent recouvrement de sa mémoire. Une fois cette mémoire recouvrée, se pose la question de son usage exemplaire. Quelle mémoire fait Bakhita des crimes qu'elle a endurés? Aurait-t-elle oublié le traumatisme de sa souffrance? Ou, en serait-elle restée à se prélasser de la nostalgie de la liberté perdue? Bakhita ne raconte pas son histoire comme les historiens de l'esclavage décrivent ces événements, de manière chronologique et phénoménale. Elle se souvient en fait de son passé pour inventer un meilleur futur. Bakhita partage le même souci que le récit évangélique originellement structuré autour de la Passion et de la résurrection. Luc écrit pour que Théophile puisse réaliser la certitude de l'enseignement qu'il a reçu (voir Lc 1,4). Jean sélectionne certains signes afin que les disciples puissent croire que Jésus est le Messie, le Fils de Dieu, et par cette croyance, qu'ils puissent avoir la vie en son nom (voir Jn 20,31). Les évangiles, comme un récit, sont un acte de mémoire qui culmine en ce que Jean Baptiste Metz (1979, 119-137) a appelé memoria passionis, un souvenir de la souffrance qui peut devenir le ferment de l'avenir de l'humanité. Ainsi, la mémoire, même dans une perspective chrétienne, n'est pas un enregistrement fidèle et intégral du passé, mais plutôt une sélection des épisodes les plus signifiants de ce passé, événements capables d'inventer un meilleur futur. Todorov (1995b, 14) dit à ce propos que la mémoire n'est pas une conservation totale du passé et qu'il y a une part d'oubli utile et intentionnelle dans tout acte de mémoire. En termes chrétiens, la mémoire doit servir la cause de l'optimisme et de l'espérance, tout comme l'eucharistie de la souffrance de Dieu, cette 
anamnèse qui célèbre la mémoire de la mort, annonce aussi la résurrection. L'idée de capital possible de rédemption est en cela corollaire de la mémoire de la souffrance. Comment Bakhita nous transmet-elle cette espérance pour une humanité meilleure?

Dans la préface à son récit, la biographe établit une excellente relation entre l'esclavage de Bakhita et l'enfance contemporaine exploitée. À dire vrai, le récit de Bakhita n'a pas de sens s'il ne nous montre pas le chemin à suivre pour faire des esclaves trafiqués aujourd'hui de possibles Bakhita. En canonisant Bakhita, le pape Jean-Paul II l'a présentée à la communauté des croyants comme "la sœur universelle ", un modèle d'imitation. Mais de quoi Bakhita est-elle vraiment le modèle universel? Un modèle de bon esclave pour les esclaves ou un modèle d'être humain pour les maîtres ? Ni l'un ni l'autre. Les catégories de l'exclusion, les esclaves contre les maîtres et inversement, ne sont pas englobantes de la mémoire de Bakhita. La mémoire de Bakhita ne doit pas non plus être manipulée par des spiritualités désincarnées qui recommandent la prière et le jeûne à des affamés. Le modèle de Bakhita réside dans ce qu'elle a été tout au long de sa vie: une esclave qui a lutté pour sa liberté. Un combat dont on saisit le sens en lisant l'histoire de sa vie à rebours.

La vie de Bakhita est un perpétuel combat contre l'oubli. Malgré le traumatisme qu'elle a enduré, elle s'est rappelé de manière très précise ce qui lui est arrivé. Si elle a pardonné à ceux qui lui ont fait du mal, elle n'a pas oublié le mal qui lui a été fait. Même si le ton de son récit est édulcoré par les expressions d'indulgence et de pardon, Bakhita y décrit sa souffrance avec les concepts mêmes du répertoire de l'esclavage. On peut reconstituer tout le champ sémantique de l'esclavage, grâce à ce vocabulaire ad hoc. Elle parle de razzia, des ravisseurs, des négriers, des maîtres et des maîtresses, des tortionnaires, des marchands d'esclaves, du marché humain, de la caravane des esclaves, des bêtes de somme, des petits nègres, de pauvres esclaves, de grosses et lourdes chaînes, de chagrin, de violence, de souffrance, d'angoisse, de fatigue, de cruauté et de brutalité, de torture et de douleur, de découragement, de tristesse, des malheurs, de faim, de mauvais traitements, de vendre et d'acheter, de donner en cadeau un être humain, etc. Tous ces maux appartiennent à la description que fait Bakhita de son esclavage, et elle évite la confusion des genres entre le souvenir et l'oubli.

Alors que l'acte chrétien de mémoire est ambivalent, le souvenir de la mort et de la résurrection (voir 2Th 2,11-13), mal et bien qui sont en fait 
les deux faces de toute alternative historique, les chrétiens n'aiment pas se rappeler des aspects négatifs de leur passé. Les chaînes de l'esclavage des Noirs perturbent la conscience chrétienne qui n'aime pas en faire mention (Maxwell 1975). De curieux interprètes de la Bible, empruntant aux ethnologues païens, ont démontré que l'esclavage des Noirs était la volonté du Dieu des chrétiens (par exemple Boesak 1977, 25-33; Messina 1996, 183 199). Comme Herbert Marcuse $(1968,123)$ l'a dit, la mémoire du passé peut rappeler des notions dangereuses, des contenus subversifs que l'on redoute. Au commencement de la traite atlantique était Bartolomé de Las Casas qui suggéra l'idée de l'asservissement des Noirs pour alléger la souffrance des pauvres Indiens ${ }^{4}$. Et même avant de Las Casas, le pape Nicholas $\mathrm{V}$, en 1454, autorisait le Portugal à pratiquer l'esclavage à la fin de l'évangélisation (Sala-Molins 1987, 60). En fait, comme conclut Nooman (1993, 664), il fut un temps, jusqu'en 1860, où l'Église enseignait que ce n'était pas un péché pour un catholique de posséder un autre être humain. Pendant la traite, l'Église catholique recevait ses dividendes de la traite des Noirs; et il y avait des missionnaires qui possédaient leurs propres esclaves. Très peu pouvaient dire qu'ils étaient comme Martin de Pores, l'esclave des esclaves.

Le regard chrétien sur l'esclavage n'a pas fondamentalement changé. Le trafic des enfants, par exemple, ne choque pas vraiment la conscience des Églises locales comme corps. Tout comme les leaders chrétiens coloniaux ont compris l'esclavage au nom de motifs économiques, des clercs locaux tolèrent le statu quo au nom de raisons culturelles. Excepté quelques engagements privés et charismatiques en faveur des victimes, il n'y a pas vraiment des communiqués officiels condamnant le trafic des enfants comme un esclavage qui doit être combattu, comme le suggérerait Bakhita à l'Église qui se targue d'être fière de sa mémoire. Certes, des évêques africains, délégués du Symposium des Conférences épiscopales d'Afrique et de Madagascar, en pèlerinage à la Maison des esclaves de Gorée, au Sénégal,

4. Sujet de grande controverse dans la reconstitution de la vérité historique au sujet des origines chrétiennes de la traite atlantique. Pour certains, Las Casas ne s'est pas seulement contenté de dénoncer le trafic des Noirs, mais il a aussi cherché à les protéger. Pour les autres, l'évêque de Chiapas a plutôt une option sélective de la dignité humaine, les bozales (les Noirs) étant différents des ladinos (les Indiens). Sur les premiers, voir Pérez-Fernandez 1991; Sullivan 1955 et sur les autres, Capdevila 1998; Quenum 1993. De toutes les manières, il n'y a pas de doute que l'Église catholique a tout au moins toléré les politiques esclavagistes, tout comme d'autres Églises chrétiennes, plus récemment, ont proposé une théologie raciste pour supporter l'apartheid en Afrique du Sud. 
le 5 octobre 2003, ont demandé pardon à l'Afrique pour la traite des esclaves à laquelle des Africains auraient participé. Mieux, ils ont recommandé aux dirigeants africains de "condamner les différentes formes de traite et d'esclavage que sont la déportation de nos filles pour la prostitution, le tourisme dit sexuel, le commerce d'enfants, l'enrôlement de force de nos enfants et adolescents dans les guerres fratricides, néocoloniales et de pillage des richesses des sous-sols africains" (Documentation catholique 2003, no 2301, 969). Jean-Claude Djéréké (2005, 182-188) offre une critique intéressante de ce "pardon de l'Afrique à l'Afrique » des évêques africains qu'il qualifie, à la suite des historiens africains, de dédouanement facile.

Ce qui est grave, c'est qu'il y a des clercs qui, aujourd'hui encore en Afrique, "possèdent » des petits boys qu'ils affectent à la production de leurs biens, et certains de leurs leaders ne se distinguent pas des esclavagistes quant à la manière dont ils traitent ceux qui sont sous leur autorité. Au Bénin, par exemple, des clercs comme les cadres ont leurs vidomegon - ces petits, pour la plupart d'origine rurale, transférés en ville auprès d'un parent dans le meilleur des cas. Ils sont corvéables à merci, les premiers à se réveiller le matin et les derniers à se coucher le soir. Comme le remarquent Chabal et Daloz, les responsables de la maltraitance n'acceptent pas les accusations: "Montrés du doigt par des associations internationales critiquant la surexploitation de fillettes à Cotonou pour servir de bonnes à tout faire, des chefs de famille béninois se sont ainsi empressés de déclarer que ces organismes étrangers "ne comprennent rien à l'Afrique" " $(1999,161)$. Le problème est que même ceux qui sont nourris des valeurs de l'Évangile trouvent cet assujettissement culturellement acceptable. Dans ces conditions, on conviendra qu'il est difficile pour les prêtres de prêcher contre les formes actuelles de l'esclavage. Le mieux qu'ils puissent faire est de recommander dans leurs sermons aux maîtres d'être gentils avec les victimes. Par exemple, suivant l'affaire Itinero (Anti-Slavery 2001), du nom du bateau transportant des enfants trafiqués et retournés à Cotonou, l'archevêque béninois a écrit une lettre dans laquelle il n'identifiait pas le phénomène du trafic des enfants comme un esclavage (La Croix du Bénin 2001). Ce faisant, il a endossé la perspective des responsables politiques et de certains intellectuels de ce pays qui abhorrent parler de ce trafic comme d'un esclavage. Dans sa "gentille» lettre, Mgr Assogba rappelait tout le bien que son diocèse faisait en faveur des enfants en situation difficile et en appelait à la générosité de sa communauté pour aider les 112 victimes. Il termina sa lettre par les mots du Seigneur appelant à recevoir les enfants en 
son nom (Mc 9,37). L'esclavage et ses survivances exigent plus que de la charité religieuse. C'est un crime dont il faut combattre les structures par un engagement clair en faveur de la justice. Et établir une société plus juste est tout l'enjeu de la récupération de la mémoire du crime.

La leçon de Bakhita est que l'enjeu du recouvrement de la mémoire ne se situe pas en termes alternatifs de pardon (ou d'oubli) et de justice (ou de souvenir), mais dans une articulation judicieuse des deux afin que ce qui est rappelé soit utilisé pour la justice. Au terme, il s'agit d'un processus de réconciliation avec soi-même (la victime) et avec l'autre (le bourreau). Voilà pourquoi l'éthique de la mémoire a une double exigence: une exigence visà-vis de soi-même et une exigence vis-à-vis des autres (Grosser 1989, 244), et non le privilège d'utiliser la souffrance du passé comme source de pouvoirs et de faveurs (Steelle 1991) comme le signifieraient les demandes de réparations économiques. La mémoire, dit saint Augustin (1964, 269), est le présent du passé, la lecture du passé à partir du présent; et Michel de Certeau $(1973,159)$ a raison d'assigner à l'histoire le rôle de la définition d'un nouveau présent, par une praxis qui transforme les traditions et change les légendes. On comprend pourquoi la même Bakhita qui se dit prête à baiser les mains de ses négriers de ravisseurs (98) a aussi martelé à la fin de sa vie de desserrer les chaînes de l'esclavage.

Mais chez Bakhita, la réconciliation déplace le niveau de justice de l'acte de mémoire, et l'enracine en Dieu. À ce niveau, la réponse devient spirituelle, même si l'acte de réconciliation peut aussi se situer dans l'anthropologie de la plénitude existentielle. La communion est toujours possible entre les humains, malgré leurs différences (Todorov 1995a, 168-169). Cette réconciliation doit être bilatérale: le pardon donné doit être acceptable et accepté, capable de créer la communion des cœurs. Bakhita n'a pas fui ses maîtres italiens, parce qu'ils étaient bons pour elle, et dans cette humanité partagée, elle était capable de donner son amour. Cette réconciliation devient impossible lorsque les deux parties ne se considèrent pas dans une relation de différence complémentaire. Or, même une équitable lex talionis reste une forme primaire de l'acte de mémoire qui n'est pas encore arrivé à faire le tri salutaire entre le souvenir et l'oubli. Le passé du crime doit être connu contre son effacement, mais il doit aussi être orienté vers l'invention du meilleur futur possible. Bakhita nous donne l'exemple de cet usage du passé créateur d'avenir. 


\section{La récupération: hagiographie et inculturation}

En élevant Bakhita au rang des saints et saintes, l'Église l'a proposée comme un modèle universel digne d'imitation. Les chrétiens sont appelés à rendre vivante la mémoire de son esclavage. Mais dans un contexte de survivances esclavagistes, l'on est en droit de se demander à quelle cause sert la récupération de cette mémoire si elle ne desserre pas les chaînes contemporaines de l'esclavage. Je voudrais suggérer une récupération en vue de l'inculturation, c'est-à-dire d'une authentique reprise africaine du message de l'Évangile tel que Bakhita l'a cru. Il va sans dire qu'il y a une fausse et obsolète mémoire qui ne sert pas la cause de Bakhita. Pour se détourner de ces mauvais usages de la mémoire, la simple question que le chrétien pourrait se poser en situation d'assujettissement consiste à savoir ce qu'aurait fait Bakhita si elle avait été là. Qu'aurait fait Bakhita devant le phénomène du trafic et du travail des enfants par exemple? La réponse à cette question ne devrait pas se limiter à une pieuse invocation de sainte Bakhita. On ne devrait pas en rester aux neuvaines de la piété, flatteuses des âmes africaines qui peuvent enfin se recommander à l'intersession d'une sainte autochtone qui compte parmi les rares dévotes que l'Église africaine a connues. Le panégyrique classique consiste à célébrer l'exceptionnelle fille du Soudan, la seule Africaine jamais canonisée, excepté les martyrs des premiers siècles chrétiens. Le plus important n'est pas l'honneur et la fierté qu'elle offre au christianisme africain. Encore qu'il n'y ait pas, à vrai dire, une fierté à rappeler l'esclavage des chrétiens! La récupération de l'esclavage de Bakhita est comme une eucharistie au sortir duquel nous retournons au monde pour délier les chaînes de l'esclavage.

Comme une leçon d'hagiographie, Bakhita est un modèle d'inculturation de la foi chrétienne qui est très différent des belles théories d'authenticité, parce que sa vie est une théologie et la théologie qu'elle enseigne est témoignée par sa vie. En cela même, son modèle d'inculturation de la foi constitue "un principe d'inspiration» (Azevedo 1992). Et c'est Dieu luimême qui affirme le principe d'inculturation en communiquant sa rédemption par l'histoire, l'histoire d'un salut par l'historicité de la culture quotidienne: l'Emmanuel sur les chemins ordinaires de la vie. Saint Paul a très vite compris cette sécularisation nécessaire de la parole de Dieu, en recommandant l'ouverture des portes de l'Église aux Grecs, aux femmes et aux esclaves, et en proposant ces catégories de l'exclusion comme possibles modèles du christianisme en devenir, non seulement en termes de dispositions à recevoir la parole de Dieu, mais aussi comme la disponibilité de 
celle-ci à rencontrer la parole de l'homme. Le christianisme lui-même devient ainsi une culture qui ne rencontre l'homme et la femme que dans leur culture; en termes africains, le christianisme et l'Afrique deviennent une chance réciproque (Kabasele-Lumbala 1993). La relation entre inculturation et hagiographie est causale, la dernière étant une réponse culturelle à la première. Et lorsque le pape Jean-Paul II demande aux Églises africaines de rédiger leur propre martyrologie $(1995,34)$, seule une sérieuse et non folklorique inculturation peut répondre à cet appel.

L'inculturation implique la reconnaissance et l'appréciation des droits de toute culture à proclamer Dieu et à transmettre son Évangile avec les catégories séculières de ce peuple - catégories de l'esclavage et de libération pour le peuple de Bakhita. Elle tient d'un principe, pas seulement pour l'évangélisation, mais aussi pour l'Évangile et, comme tel, on en saisit l'impératif. Ne pas l'admettre c'est contredire le but même de l'Évangile qui est l'avancement du règne de la paix, et a contrario tolérer la progression du règne de la violence occasionnée par les régimes de l'esclavage. Ce n'est pas surprenant que le christianisme colonial n'a pas tenu ce principe en vue de l'évangélisation des Noirs. Ainsi, la richesse de l'Évangile a été appauvrie par des théologies dites d'adaptation (Des prêtres noirs s'interrogent 1956). Comment les missionnaires pouvaient-ils poser l'inculturation comme principe lorsque l'évangélisation consistait d'abord en une mission civilisatrice de ceux qui n'avaient pas de culture? Partout où l'esclavage a pris le pas sur la liberté, la procuration a substitué l'inculturation. Les Églises sous tutelle devaient imiter les Églises mères et les saints et saintes des maîtres étaient aussi les modèles des esclaves. Avec Bakhita, la page de l'imitation servile est tournée et l'hagiographie africaine vient au secours de la théologie. On revient à l'argument de la biographie comme théologie (McClendon 1970), dans la mesure où la théologie, comme une diction de Dieu, a pour rôle de donner sens aux pratiques culturelles des hommes et des femmes. À partir du récit africain, Bakhita nous enseigne qu'une théologie africaine peut commencer par la proposition de modèles de libération de l'esclavage.

Africaine, Bakhita n'est pas une figure étrangère comme l'étaient les modèles coloniaux proposés aux indigènes. Bakhita est pertinente pour l'Afrique, parce qu'elle est une réponse africaine au christianisme. Mais la connaître n'est pas assez: il faut aussi répondre à son appel de la lutte titanesque contre les derniers bastions de l'esclavage caractérisés par les formes de paupérisation anthropologique toujours présentes, triomphantes et 
arrogantes (Mveng 1992, 111-119). Bakhita nous dit que la vie est encore possible en Afrique, et que l'histoire de l'esclavage, pour cruelle qu'elle soit, ne peut épuiser le réservoir de grâces enfouies dans la culture africaine qui est une spiritualité de vie en abondance (Magessa 1998). En cela précisément, la spiritualité de la nouvelle évangélisation que propose Marcello de Azevedo (1995) et qui est incarnation-inculturation, salutlibération et communication-communion (109-118), est témoignée par le modèle de Bakhita qui s'élève ainsi de l'Afrique et embrasse le monde des chrétiens. Trois étapes dialectiques peuvent caractériser ce processus de récupération de la mémoire de Bakhita: la dénonciation de la théologie de l'esclavage, l'énonciation d'une théologie de la liberté et l'annonciation d'une théologie de la rédemption.

La première chose à faire consiste à dénoncer les formes coloniales du christianisme qui sont toujours à l'œuvre en Afrique, dans la continuation de l'héritage colonial de la violence au nom de Dieu. Il ne s'agira plus d'un procès du christianisme colonial en tant que tel, mais de ses survivances contemporaines. Bakhita ne peut se sentir à l'aise dans une Église qui ne s'engage pas à combattre ouvertement et prophétiquement le trafic des enfants et les structures du déni de droits humains. Bakhita ne peut être en communion avec une Église autocratique qui a une conception sélective de la dignité humaine et qui marginalise les femmes. Les droits humains fondamentaux doivent pouvoir être intégrés dans les commandements de Dieu et de l'Église, et partout où ils sont niés, la mémoire de Bakhita oblige à protester. Tel est l'impératif des chaînes de l'esclavage qu'il faut délier.

Une fois offertes les conditions minimales de justice pour tous, une théologie d'énonciation du message peut enseigner aux Africains leurs différentes manières, comme anciens esclaves ou esclaves potentiels, de récupérer la mémoire de Bakhita en devenant des chrétiens dont les témoignages de vie rappellent la mémoire de réconciliation de Bakhita libre. La réconciliation doit être le mot d'ordre d'un Évangile africain. Les Africains ont besoin de se réconcilier avec eux-mêmes et avec ceux qui les ont réduits en esclavage et leur ont appris à perpétuer la logique infernale de la loi du plus fort. Énoncer l'Évangile en termes de réconciliation serait salutaire pour l'Afrique des divisions tribales et ethniques, des ségrégations économiques et sociales. Bakhita nous apprend que la réconciliation est possible entre l'esclave et le maître, pourvu que chacun reconnaisse la dignité de l'autre. Bakhita est un modèle opératoire de la théologie après le génocide rwandais, les nettoyages ethniques et les manipulations tribales ici et là en 
Afrique. L'importance de cette réconciliation indispensable à la vie en société est dévoilée au troisième niveau du fait de la prééminence des valeurs du royaume.

Bakhita a pardonné à ses ravisseurs et tortionnaires pour des raisons qui ne peuvent pas seulement être élucidées par le besoin de la coexistence sociale. La raison fondamentale pour laquelle elle peut s'agenouiller pour baiser les mains des criminels (98) est qu'elle ne serait pas devenue chrétienne et religieuse, sans "l'heureuse faute». Nous sommes ici de plainpied dans la foi que rien ne peut ébranler. Une théologie suspensive d'annonciation du règne à venir apparaît chez Bakhita et est révélée par la profonde conviction qui soutient sa vie et ses actes. Mais cette foi, en anticipation de la récapitulation finale d'une histoire affectée par le péché, est déjà actuelle et présente chez Bakhita, comme la joie et la douceur de sa relation pacifiée avec son entourage le montrent. Par exemple, Bakhita s'entiche de la petite fille des Michieli (55), son maître italien, au point que, lorsqu'elles devraient se séparer, la petite fille ne voudrait plus la lâcher (60). Au couvent, c'est tout le monde qui veut être l'ami de Bakhita: les jeunes filles de l'école où elle était portière (82), les ouvriers de la filature de laine qui la considéraient comme leur mère (85), les humbles gens et les personnes de haute classe (88). Le traumatisme de l'esclavage est alors minimisé par l'espérance d'une humanité enfin réconciliée. Dans les situations limites de mort que vit l'Afrique, Bakhita nous apprend, comme dit Marie dans le récit de l'Annonciation, que rien n'est impossible à Dieu (voir Lc 1,38).

Si l'inculturation présuppose la reprise du christianisme par les symboles culturels, alors le paradigme de Bakhita offre ce cadre de récupération du christianisme africain. L'hagiographie dans les Églises africaines, dans une restriction à l'onomastique liturgique, s'est contentée de célébrations joyeuses et colorées. Les Africains peuvent apprendre beaucoup plus de leurs saints et saintes, surtout ceux et celles qui exigent une conversion radicale. À voir le succès qu'ont les bienheureux aux vertus sexuelles comme Anuarite Nengapeta ou les mystiques comme Isidore Bakandja, du fait de leur héroïsme qui appartient au témoignage privé, on peut comprendre que l'appel de Bakhita à un engagement public est plus difficile à entendre. En fait, Bakhita est une «sainte politique », dans la mesure où elle confronte les mœurs de la cité assiégée, et peut alors être proposée comme modèle de la reconstruction sociale de l'Afrique. Accepter son appel à desserrer les chaînes de l'esclavage, c'est s'engager pour la justice et la paix 
partout où la liberté humaine est menacée. Une théologie consciente des tragédies africaines et intéressée par le modèle de Bakhita pourrait élaborer une mémoire africaine de l'esclavage comme possible capital de rédemption.

\section{Le possible capital de rédemption}

Pour comprendre l'impératif d'un usage rédempteur de la mémoire de l'esclavage il faut commencer par rappeler l'actualité du principe au nom duquel les autres se font l'impérieux devoir de sauver le Noir: l'Afrique est toujours «enveloppée dans la couleur noire de la nuit» (Hegel 1964). Aussi, les observateurs et les experts de l'Afrique ont renoncé à l'espoir d'un jour meilleur pour le continent noir. Plus grave encore, des néohégéliens ont repris du service pour prédire l'inéluctable disparition du continent de la damnation historique, comme conséquence de ses malheurs fatals que la raison humaine ne peut plus prévenir. Et à dire vrai, l'Afrique de la mort banale ou de la banalité de la vie confirme la littérature de l'apocalypse. Putatifs descendants de Cham (Gn 9,18-27), une certaine théologie les a identifiés comme les maudits des dieux bibliques (Koffi 2000). Parias de l'espèce humaine (Hadjadj 1998), que ce soit en Afrique ou ailleurs, les Noirs sont considérés comme la risée du monde. Tout se passe encore comme s'il y avait un profil historique qui sert toujours de mesure d'identification des Noirs, qu'ils soient de la Nouvelle-Orléans ou du Darfour: lorsque survient le malheur, un ouragan ici et une guerre là-bas, la précarité de leur vie est dévoilée; et être Américain ou Soudanais est simplement un voile identitaire qui cache le Noir de partout. L'abjection est si forte que, si la question de leur humanité n'est plus explicitement exprimée, la réalité du racisme comme «un système de la suprématie et de la supériorité des Blancs» (Guider 2001) n'a pas disparu.

Contre les «prévisions savantes» d'une Afrique abjecte, il y en a qui pensent qu'une renaissance africaine est possible. Paradoxalement, l'Afrique est vue comme le miroir du monde qui renverrait à celui-ci ses ambivalentes faces (Enghelard 1998) et d'où pourrait surgir le nouvel homme mondial (Enghelard 1996). Dans ce contexte de subversion de perception, l'esclavage peut conduire à la liberté. C'est cette signification positive de la métaphore paulinienne de l'esclavage qui est ainsi retrouvée par Bakhita: l'esclave du Christ, mais davantage esclave comme le Christ, le maître qui lave les pieds de ses disciples. Dale Martin (1990) voit dans cette perspective l'esclavage comme salut, sous certaines conditions, et c'en sont les modalités africaines que le concept de possible capital de rédemption 
explore. Du fait de leur histoire, les Africains ont un atout extraordinaire de réinterprétation de l'économie du salut en contexte contemporain de mondialisation, de sorte que la rédemption puisse être disponible pour tous. La vie de Bakhita, hommage à la béatitude de la pauvreté, témoigne de cette grâce d'être pauvre. Il n'y a pas de souffrance humaine plus forte que la grâce de Dieu. Le possible capital de rédemption est une réponse qui permet de transformer l'esclavage en une liberté qui fructifierait les grâces qui nous sont données en capital capable de réaliser notre rédemption - saint Paul parle à ce propos des arrhes de l'Esprit (2Co 1,22; 5,5). De quoi ce capital est-il constitué ? À quelles conditions ses «actions» sontelles productives? Le modèle de Bakhita aide à répondre à ces questions.

La littérature du développement moderne tient la culture africaine comme la cause première du malheur du continent. À ce propos, le traité de David Landes (1998) sur la richesse et la pauvreté des nations, explicite en termes de défaillantes valeurs culturelles ce que certains Africains euxmêmes ont appelé le refus du développement (Kabou 1991) ou le besoin d'un programme d'ajustement culturel (Etounga-Manguelé 1991). Mais pour paraphraser ce dernier, c'est davantage d'un projet d'ajustement historique, par un usage judicieux de la mémoire de l'esclavage, qu'il s'agit. C'est surtout l'histoire des esclavages, d'hier et d'aujourd'hui, qui est à la base du malheur africain. La géographie des guerres africaines se situe aux confins des productions des matières premières congolaises, angolaises, sierra-léonaises, libériennes, soudanaises, etc. La plupart des pays africains à la une des rapports sur la pauvreté dans le monde ne sont pas des pays pauvres, mais plutôt des pays appauvris par des mécanismes toujours renouvelés de paupérisation perpétuelle. Si l'on ne renonce pas aux discours de complaisance des Africains et de condescendance des Occidentaux au sujet d'une pauvre Afrique qu'il faut aider, alors l'hémorragie anthropologique du Noir peut conduire vraiment à sa mort. Seule une mémoire juste de l'esclavage peut suspendre le temps fatal de la croix et remettre l'Afrique sur la trajectoire d'un vendredi saint nécessaire au matin de Pâques. Jésus a fait valoir la croix au point d'en faire la condition de sa suite pour entrer dans le royaume de Dieu (voir Mc 8,34-36). Mais l'acceptation de la croix et de la souffrance ne répond pas seulement à une nécessité historique; l'enjeu est autant ontologique. Saint Paul développe sa théologie de la kénose de ce fait (voir Ph 2,6-11). Si un prix Nobel de la kénose historique des peuples devait être décerné, il reviendrait aux Noirs dont les esclavages ont enrichi les autres. De ce fait, ils forment une particulière communauté de souffrance avec l'homme de la croix. Si ce dernier a pu vaincre la mort, 
il n'y a pas de raison que les Noirs qui se réclament de sa mémoire ne retrouvent pas la vie. Je propose trois conditions nécessaires à l'avènement de la vie en Afrique noire, modalités qui participent de la réinvention du sens de la croix africaine. Ce sont: (1) la subversion du récit de l'esclavage, (2) une rupture historique et épistémologique de l'interprétation de ce récit et (3) une rébellion prophétique.

L'élaboration systématique du discours occidental sur l'esclavage congénital des Noirs trouve son origine dans le présupposé hégélien du continent des ténèbres et du chaos à la recherche d'un salut et d'une lumière qui ne pouvait venir que de l'Occident. Reprenant Hegel, le discours fondamentaliste de la fin de l'histoire accomplie par l'économie de marché néolibérale (Fukuyama 1992) réaffirme la nécessité de l'expansion de la liberté chez les nouveaux barbares qu'il faut démocratiser. Ainsi des guerres actuelles de l'Occident en Afrique par des bandes ethniques de nègres de service interposés. Le paradigme néolibéral, nouvel évangile de l'Occident sécularisé, est incapable d'affranchir l'esclave. Si la mission civilisatrice d'hier n'a pas pu «civiliser» le Noir, ce n'est pas la mission humanitaire d'aujourd'hui qui pourrait "l'humaniser». Et on connaît les affres de la mondialisation de l'économie qui a consacré la marginalisation de l'Afrique noire. Il faut forcément sortir des labyrinthes de ce nouveau désordre mondial (Todorov 2003) du déterminisme historique et ouvrir l'histoire de l'esclavage des Noirs aux possibilités endogènes de rédemption.

Une possibilité d'interprétation de cette mémoire est de prendre à rebours le sens que le maître donne à l'histoire de l'esclave. Le travail de réinterprétation du discours chrétien et occidental sur l'Afrique doit partir des Africains qui veulent "sauver» l'Afrique (Diallo 1996), à partir du sens qu'ils donnent à leur croix. Une praxis de l'inculturation à la Bakhita, comme précédemment exposée, reviendrait à partir des portraits africains de dignité. Pour Bakhita, il s'agit de la fière dignité d'être ce qu'on est; une valeur qui manque aujourd'hui à beaucoup d'Africains dont l'imitation servile du maître devenu modèle a pris des proportions inquiétantes. Ce projet de re-lecture de l'esclavage (Paulo Freire) devrait conduire à l'invention d'un nouveau monde de liberté qui interprète l'esclavage des Noirs en catégories de salut et non sous l'ancien modèle de vide qu'il faut combler. Les Noirs n'ont aucun vide ontologique à combler, mais plutôt un espace théologique à remplir. Et voici la nuance: une chose est de se faire à l'image des autres, autre, de Dieu. D'où la difficile question: Dieu peut-il se reconnaître dans l'image de l'esclave africain? Pour saint Paul, pour qui Dieu choisit ce qui est faible et que l'on méprise dans le monde pour confondre 
les sages et les forts (voir 1Co 1,26-31), le Dieu de Jésus est celui de «la condition d'esclave» (Ph 2,6). C'est cette communauté dans la souffrance avec le crucifié qui confère aux Africains le droit d'une lecture autonome et d'une appropriation de l'économie du salut; une prérogative qui supplanterait ainsi les énonciations hétéronomes de la parole de Dieu. Certes, la souffrance n'est pas une réalité quantifiable, et une mère qui perd son enfant ne souffre pas moins que celle qui en perd dix. Mais du point de vue historique, dans l'espace et dans le temps, comme le dit l'historien britannique John Ilife que cite l'économiste français Engelhard (1998), «la souffrance se trouve au cœur de l'expérience africaine et semble l'emporter sur celle des autres continents» (30). Il ne revient plus aux autres de dire aux Noirs qui est Dieu, car la théologie du maître ne peut plus être celle de l'esclave. Il s'agit donc non seulement d'une question d'émancipation en termes de cassure du discours, mais davantage d'autodétermination.

L'appropriation du discours conduit à la deuxième condition. Déboussolés, les Africains se demandent d'où viendra leur salut. Le salut ne viendra pas de la campagne mondiale pour l'éradication de la pauvreté ou de l'effacement de la dette, pour ne citer que les propositions de panacées les plus spectaculaires. Il faut plutôt s'intéresser aux sources de génération de cette pauvreté endémique qui remontent aussi bien à la méconnaissance des structures de l'esclavage qu'à sa mauvaise interprétation. Dans le cadre d'une historiographie africaine, Cheikh Anta Diop $(1954,14$; 1967, 26) a suggéré des ruptures historique et épistémologique qui devraient rétablir l'antériorité des civilisations nègres contre la falsification idéologique par une tabula rasa des vérités et dogmes de l'Occident. À cela, j’ajoute le refus du discours de complaisance aux Africains. Simplement dit, cela reviendrait à définir l'esclavage et appeler, par exemple, le trafic et le travail des enfants une survivance de cet esclavage. Diop $(1981,12)$ recommande de tenir les deux principes de continuité historique de créations culturelles des sociétés africaines, et l'unité des peuples et des civilisations noires, par un retour à l'Égypte.

Le retour à l'Égypte pharaonique, comme point d'origine du récit africain, ou au Soudan dans le cas de Bakhita, est une rupture qui remonterait l'historicité de l'esclave dans le temps et le situerait dans son espace de création de valeur et de sens. Contrairement aux perceptions d'une Afrique spontanée et imaginaire qui conduit inéluctablement au désespoir, l'identité du Noir n'a pas toujours été celle de l'esclave. Bakhita n'est pas née esclave. Elle a toujours gardé une mémoire vivante du passé de son enfance avant son enlèvement malgré les traumatismes subis. Elle savait qu'avant son 
esclavage, elle avait un père, une mère, des frères et sœurs très aimants et une famille très affectueuse dans sa communauté tribale (Jones 1999, 188) avant son épopée religieuse avec les chrétiens. Dans son propre récit, elle parle de son village d'Afrique avec une nostalgie enjouée que même son paradis italien n'est pas arrivé à faire oublier. En n'oubliant pas la liberté dont elle jouissait avant son esclavage, Bakhita était assez forte pour lutter pour le recouvrement de cette liberté confisquée. Bakhita ne s'est jamais résignée à accepter le statu quo: elle a fui (41-44), désobéi, protesté même si elle a dû être punie par les deux dames du général turc (46-48). Par une rupture avec le passé du mal, les valeurs rédemptrices du présent pourraient être trouvées dans le souvenir du bonheur perdu. Cette conviction mobilisatrice peut détruire le déterminisme fatal de l'afropessimisme; d'où le troisième élément de la triade.

L'esclave ne peut se débarrasser de ses chaînes que par une rébellion. Une rébellion qu'on qualifierait de prophétique, non seulement pour la situer dans une perspective religieuse où la prophétie a pour rôle de rappeler les clauses de l'alliance à une royauté infidèle, mais davantage pour démarquer cette forme de dissidence des usages de la force qui s'en réclament. Même si l'idée de rébellion prophétique est difficile à définir dans un contexte hors-jeu où la violence appelle la violence, on peut en comprendre le sens à l'aide des exemples que Jésus et Bakhita nous donnent. Les récits de la Passion nous renseignent sur le comportement de Jésus devant ses ennemis. Il n'a pas tendu, par exemple, l'autre joue à celui qui l'a giflé (voir Mt 5,39; Jn 18,22). Voilà un exemple de rébellion, en situation de crise, qui suspend le devoir d'obéir aux ordres établis. Il est temps que l'Africain qui a toujours obéi de force aux autorités scélérates commence par désobéir de gré.

La vie de Bakhita est jalonnée de ces formes de résistances simples et ordinaires. Si elle accepte de prendre sa croix, c'est aussi par amour, amour pour les personnes, les bonnes et les mauvaises. Il y a une méprise dans l'éloge inconditionnelle que fait Maria Luisa Dagnino, sa biographe, de son obéissance. Jugeons: "Bakhita accomplit toujours ses devoirs d'esclave, tâchant d'obéir aux ordres qu'elle recevait. Son esclavage commença justement par un acte de prompte obéissance envers ses ravisseurs euxmêmes» (67). Plus loin, la biographe pour qui le destin de Bakhita était tracé (45) nous donne sa grille d'interprétation: «Il faut d'abord souligner une raison de fond: tous les malheurs, les contretemps, les erreurs, les instincts brutaux et égoïstes, les motivations vénales et commodes, tout cela concourut à la réalisation des plans de la divine providence» (91). On 
trouverait à redire sur cette interprétation déterministe de l'esclavage de Bakhita dans la mesure où elle a combattu pour le recouvrement de sa liberté avec les moyens naturels dont elle disposait - sa parole et sa volonté. Un bel exemple nous est donné par Bakhita lorsque, voulant faire valoir ses droits sur elle, sa patronne, $\mathrm{M}^{\mathrm{me}}$ Turina, la contraignit de retourner avec elle en Afrique (59). Bakhita dit simplement non et accepta d'être traitée d'ingrate. Dire non aux chaînes de l'esclavage, voilà en quoi consiste une rébellion prophétique. Même la prétendue volonté de Dieu s'éclipse devant la liberté humaine; raison pour laquelle l'homme a toujours la possibilité de se rebeller contre la plus orthodoxe interprétation de la volonté de Dieu. Il est temps de se rebeller contre les pratiques d'une théologie, dans l'Église et dans le monde, qui, hier a interprété l'esclavage des Noirs comme la volonté du Dieu des chrétiens et des arabes, et aujourd'hui, le libéralisme économique comme le seul chemin du bien-être vers la liberté des anciens esclaves en quête de la liberté.

\section{Conclusion}

Suivant l'appel de Paul à se souvenir de Jésus Christ (voir 2Th 2,3-12), dans sa souffrance comme dans sa résurrection, ces pages ont essayé de proposer un usage de la mémoire africaine de l'esclavage comme un possible capital de rédemption. Pour y parvenir, trois points ont articulé une reprise africaine de cette mémoire, selon le paradigme de la vie de Bakhita. Ce sont: le récit de l'esclavage à subvertir, la mémoire à en faire, puis sa récupération dans un projet d'inculturation. Conséquemment, l'idée de possible capital de rédemption a suivi les mêmes caractéristiques: la subversion du discours chrétien par un nouveau récit, la rupture historique et épistémologique comme solution de remplacement à une mémoire obsolète de l'esclavage, et enfin une rébellion prophétique qui permet le recouvrement de la parole créatrice. Toutefois, ces éléments ne sont pas exhaustifs dans le processus de capitalisation de la mémoire de l'esclavage; ils en suggèrent seulement des usages.

Seule la parole échangée et acceptée, beaucoup plus que le pardon unilatéral et parfois forcé, pourra amener à fermer la page douloureuse de l'esclavage des uns par les autres. Peut-être aussi que c'est là que réside le problème majeur de l'Afrique: acculés, les Africains ont renoncé à la possibilité de dire non aux chantages d'un développement économique qui doit se faire au dépend de leur liberté. Cela étant, je propose finalement une seule chose: que les Noirs s'asseyent calmement pour se parler et 
s'écouter! Non plus sous les doux ombrages des palabres de l'accusation de l'absent, ou sous les hangars berceurs et enchanteurs de l'écoute de la parole d'un Dieu transitaire qui ne s'arrête jamais en Afrique, mais se contente d'y envoyer son message de compassion et ses messagers compatissants. Qu'ils s'asseyent, face à face, pour se regarder, se parler et accepter de ne pas se mettre d'accord. Le salut de l'Afrique n'est pas si loin qu'on le pense: il est en Afrique, à portée de main des Africains dont il faut seulement délier les chaînes si lourdes de l'esclavage pour qu'ils aillent à sa recherche.

L'Afrique arrive et son futur ne doit plus être son passé. Avec Bakhita et le peuple des esclaves portant la croix au devant, en suivant leur modèle, tous pourront entrer dans le royaume de liberté. Bakhita a proposé de s'agenouiller devant ses négriers pour baiser leurs mains, devrait-elle les rencontrer (98). Jésus a lavé les pieds de ses disciples. La leçon est: «Je vous ai donné un exemple pour que vous agissiez comme j’ai agi envers vous» (Jn 13,15). L'eucharistie du lavement des pieds ou le baiser des mains des trafiquants d'esclave est une subversion de rôles, une transposition de sens: l'esclave est en fait le maître. Ce faisant, Jésus et Bakhita nous enseignent que l'esclavage trouve son sens dans l'eucharistie, le banquet du royaume, le mémorial de la rédemption chrétienne. À partir de la vie de Bakhita, une judicieuse mémoire africaine de l'esclavage peut véritablement servir la cause de la rédemption. Le mandat pathétique qu'elle donne: «Desserrez les chaînes de l'esclavage» devient ainsi un commandement eucharistique: «Faites ceci en mémoire de moi». Il y a bien un autre baptême que celui du sang de l'esclave de Césaire inscrit en exergue à cet article. Ceux et celles qui répondront à cet appel, au nom de Jésus, en souvenir de Bakhita, feront lever le jour de rédemption sur l'Afrique noire.

\section{Références}

Agossou, T., dir. (2000), Regards d'Afrique sur la maltraitance, Paris, Karthala (Questions d'enfances).

Anti-Slavery (2001), «Benin's Slave Ship's Reveals West Africa's Child Trafficking Problem»(18 avril), Anti-Slavery. Today's Fight for Tomorrow's Freedom, accessible sur le Web à <http ://www.antislavery. org/archive> (page consultée le 23 mai 2006).

Aristote (1968), Économique / texte établi par B.A. Groningen, traduit et annoté par A. Wartelle, Paris, Les Belles Lettres (Collection des Universités de France. Série grecque 185). 
Augustin (1960), La cité de Dieu. Livres XIX-XXII. Triomphe de la cité céleste / introduction et notes par G. Bardy, traduction par G. Combès, Paris, Desclée de Brouwer (CEuvres de saint Augustin, 5e série 37).

(1964), Les Confessions / traduction, préface et notes par J. Trabucco, Paris, Flammarion (Garnier-Flammarion 21).

Azevedo, M. de C. (1992), "Inculturation: Problématique », dans R. Latourelle et R. Fisichella, dir., Dictionnaire de théologie fondamentale, Montréal / Paris, Bellarmin / Cerf, p. 612-624.

- (1995), The Consecrated Life. Crossroads and Directions / trad. par G. Cook, Maryknoll, Orbis Books.

Bequele, A. et W.E. Myers (1995), First Things First in Child Labor: Eliminating Work Detrimental to Children, Geneva, UNICEF / International Labour Organization (ILO Child Labour Collection).

Berlin, I. et P. Morgan (1993), Cultivation and Culture: Labor and Shaping of Slave Life in the Americas, Charlottesville, University Press of Virginia.

Boesak, A.A. (1977), Farewell to Innocence. A Socio-Ethical Study on Black Theology and Power, New York, Orbis Books.

Capdevila, N. (1998), Las Casas. Une politique de l'humanité. L’homme et l'empire de la foi, Paris, Cerf (Passages).

CÉsaire, A. (1958), Et les chiens se taisaient, Paris, Présence Africaine.

Chabal, P. et J.P. Daloz (1999), L’Afrique est partie! Du désordre comme instrument politique, Paris, Economica.

Daddah, A. (1998), "Mauritanie, les héritiers de l'esclavage », Le monde diplomatique, 11, p. 13.

Dagnino, M.L. (1992), Bakhita raconte son histoire, Rome, Canosiane Figlie della Carita.

De Certeau, M. (1973), L’Absent de l'histoire, Paris, Mame.

De Montesquieu, C.L. (1952) [français 1748], The Spirit of Laws / traduit par T. Nugent, révisé par J.V. Prichard, Toronto, Encyclopedia Britannica (Great Books of the Western World 38).

Deschamps, H. (1971), Histoire de la traite des Noirs. De l'antiquité à nos jours, Paris, Fayard.

Des prêtres noirs s'interrogent (1956), Paris, Cerf.

Diallo, M.L. (1996), Les Africains sauveront-ils l'Afrique?, Paris, Karthala. 
Diop, B.B., O. Tobner et F.-X. Verschave (2005), Négrophobie, Paris, Les Arènes.

Diop, C.A. (1954), Nations nègres et culture. De l'antiquité négro-égyptienne aux problèmes culturels de l'Afrique noire d'aujourd'hui, Paris, Présence Africaine.

(1967), Antériorité des civilisations nègres: mythe ou vérité historique?, Paris, Présence Africaine.

(1981), Civilisation ou barbarie. Anthropologie sans complaisance, Paris, Présence Africaine.

DJÉRÉKé, J.C. (2005), Rome et les Églises d'Afrique. Propositions pour aujourd'hui et demain, Paris, L'Harmattan.

Documentation catholique (2003), $\mathrm{n}^{\circ} 2301$ (2 novembre).

Drescher, S. (1992), "The Ending of the Slave Trade and the Evolution of the European Scientific Racism », dans J.E. INIKORI et S.L. EngERman, dir., The Atlantic Slave Trade. Effects on Economies, Societies and Peoples in Africa, the Americas, and Europe, Durham / Londres, Duke University Press.

Eboussi Boulaga, F. (1968), «Le Bantou problématique ", Présence Africaine, 66, p. 4-40.

(1993), Les conférences nationales en Afrique noire: une affaire à suivre, Paris, Karthala.

- (1999), Lignes de résistance, Yaoundé, Clé.

ELA, J.M. (1998), Innovations sociales et renaissance de l'Afrique noire. Les défis du monde d'en bas, Montréal, L'Harmattan.

Engelhard, P. (1996), L’homme mondial. Les sociétés humaines peuventelles survivre?, Paris, Arléa.

_- (1998), L'Afrique, miroir du monde. Plaidoyer pour une nouvelle économie, Paris, Arléa.

Etounga-Manguele, D. (1991), L'Afrique a-t-elle besoin d'un programme d'ajustement culturel?, Ivry-Sur-Seine, Nouvelles du Sud.

Fanon, F. (1968), Les damnés de la terre, Paris, Maspero.

Freire, P. (1970), The Pedagogy of the Oppressed / trad. par M. Bergman Ramos, New York, Herder \& Herder.

(1998), Pedagogy of Hope. Relieving the Pedagogy of the Oppressed, New York, Continuum. 
Funuyama, F. (1992) [anglais 1992], La fin de l'histoire et le dernier homme I trad. par D.-A. Canal, Paris, Flammarion (Champs).

Grace, J. (1975), Domestic Slavery in West Africa, Londres, Garden City Press.

Grosser, A. (1989), Le crime et la mémoire, Paris, Flammarion (Champs).

Guider, M.E. (2001), "Moral Imagination and the Missio Ad Gentes: Redressing the Counterwitness of Racism ", CTSA Proceedings 56, p. 4969.

Hadjadj, B. (1998), Les parias de la mondialisation. L'Afrique en marge, Paris, Présence Africaine.

Hegel, G.W.F. (1964), Leçons sur la philosophie de l'histoire / trad. par J. Gibelin, Paris, Vrin.

Helene, J. (1997), «Esclaves au marché noir », Le monde (1 ${ }^{\text {er }}$ janvier), 16153, p. 9s.

JeAn-Paul II (1995), Exhortation apostolique post-synodale «Ecclesia in Africa», (14 septembre 1995), accessible en ligne à <www.vatican.va/ holy_father/john_paul_ii/apost_exhortations/documents/hf_ jp-ii_exh_14091995_ecclesia-in-africa_fr.html> (page consultée le 24 mai 2006).

Jones, K. (1999), Women Saints. Lives of Faith and Courage, Maryknoll, Orbis Books.

Kabasele-Lumbala, F. (1993), Le christianisme et l'Afrique. Une chance réciproque, Paris, Karthala.

Kaвou, A. (1991), Et si l'Afrique refusait le développement?, Paris, L'Harmattan.

Koffi, E. (2000), «Rethinking the Significance of the Black Presence in the Pentateuch for Translation and Studies Bibles. Part I», The Bible Translator, 51/3, p. 316-330.

Kunn, T. (1970), The Structure of Scientific Revolutions, Chicago, University of Chicago Press.

La Croix du Bénin (25 mai 2001).

Landes, D. (1998), The Wealth and Poverty of Nations. Why Some Are So Rich and Some So Poor?, Londres, Abacus.

Lovejoy, P.E. (1983), Transformation in Slavery. A History of Slavery in Africa, Cambridge, Cambridge University Press. 
Magessa, L. (1998), African Religion: The Moral Traditions of Abundant Life, Nairobi, Paulines.

Marcuse, H. (1968) [anglais 1964], L'homme unidimensionnel. Essai sur l'idéologie de la société industrielle avancée / traduction par M. Wittig revue par l'auteur, Paris, Minuit (Arguments).

Martin, D. (1990), Slavery as Salvation. The Metaphor of Slavery in Pauline Christianity, New Heaven / Londres, Yale University Press.

Maxwell, J.F. (1975), Slavery and the Catholic Church. The History of Catholic Teaching Concerning the Moral Legitimacy of the Institution of Slavery, Londres, Barry Rose.

Mbembe, A. (1996), La naissance du maquis dans le Sud-Cameroun (19201960). Histoire des usages de la raison en colonie, Paris, Karthala.

- (2000), De la postcolonie. Essai sur l'imagination politique dans l'Afrique contemporaine, Paris, Karthala.

McClendon, J.W. (1970), Biography as Theology: How Life Stories Can Remake Today's Theology, Nashville / New York, Abingdon.

Meillassoux, C. (1986), Anthropologie de l'esclavage: le ventre de fer et d'argent, Paris, Presses universitaires de France.

Memel-Fote, H. (1990), L'esclavage lignager et l'anthropologie des droits de l'homme, Paris, Collège de France.

Messina, J.P. (1996), «La Bible et le destin du peuple noir. Essai de réflexion critique sur le mythe de la malédiction de Cham », Mélanges de Sciences religieuses, 53/2, p. 183-199.

Metz, J.B. (1979), La foi dans l'histoire et dans la société. Essai de théologie fondamentale pratique, Paris, Cerf.

Mxveng, E. (1963), Histoire du Cameroun, Paris, Présence Africaine.

(1992), «Paupérisation et développement en Afrique», Terroirs, 1, p. 111-119.

- (1994), «Impoverishment and Liberation: A Theological Approach for Africa and the Third World », dans R. Gilbelini, dir., Paths of African Theology, Maryknoll, Orbis Books, p. 154-165.

Noonan, J.T. fils (1993), «Development in Moral Doctrine», Theological Studies, 54/4, p. 662-677.

Perez-Fernandez, I. (1991), Bartolomé de las Casas, Contra los Negros? Revisión de una leyenda, Madrid / Mexico, Mundo Negro / Esquila. 
Quenum, A. (1993), Les Églises chrétiennes et la traite atlantique du XV au $X I X^{e}$ siècle [sic], Paris, Karthala.

Sala-Molins, M. (1987), Le Code noir ou le calvaire de Canaan, Paris, Presses universitaires de France.

Samarin, W. (1989), The Black Man's Burden: African Colonial Labor on the Congo and Ubangi Rivers, 1880-1900, Boulder, Westview.

Sмith, S. (2003), Négrologie. Pourquoi l'Afrique meurt, Paris, CalmannLévy.

Steele, S. (1991) [1990], The Content of Our Character. A New Vision of Race in America, New York, Harper Perennial.

Sullivan, F.P. (1995), Indian Freedom. The Cause of Bartolome de Las Casas, 1484-1566. A Reader, Kansas City, Sheed and Ward.

Todorov, T. (1995a), La vie commune. Essai d'anthropologie générale, Paris, Seuil.

(1995b), Les abus de la mémoire, Paris, Arléa.

(2003), Le nouveau désordre mondial. Réflexions d'un Européen, Paris, Robert Laffont.

\section{Résumé}

À partir du cadre méthodologique de la biographie comme théologie (McClendon 1970), cet article propose la mémoire de l'esclavage des Noirs comme un possible capital de rédemption. L'argument est basé sur la vie de sainte Joséphine Bakhita, ancienne esclave africaine, qui, devant mourir, a supplié de desserrer les chaînes si lourdes de l'esclavage. Dans une perspective hagiographique, trois points structurent le paradigme d'une théologie de l'esclavage chez Bakhita: le récit, le souvenir et la récupération de la mémoire de l'esclavage. L'idée de possible capital de rédemption s'articule conséquemment autour des mêmes caractéristiques: la subversion du récit hégélien sur l'esclavage des Noirs par le modèle de Bakhita, une rupture historique et épistémologique de l'interprétation de ce récit, et une rébellion prophétique par la possibilité de dire non.

\section{Abstract}

Following McClendon's (1970) framework of biography as theology, this paper proposes the memory of the slavery of Blacks as possible capital of redemption. The argument is based on the life story of Saint Josephine Bakhita, a former African slave, who urged at the end of her life to loosen the heavy chains of 
slavery. In a hagiographical perspective, three steps structure this claim: the narrative of slavery, its memory, and its retrieval, each time shaped by Bakhita's theology of slavery set as paradigm. Consequently, the idea of possible capital of redemption followed the same patterns: the subversion of the Hegelian discourse by a new narrative Bakhita-based, the historical and epistemological rupture as alternative to the misleading memory of slavery, and the prophetic rebellion by the possibility to say no. 